\title{
Factores que determinan la resiliencia socio-ecológica para la alta montaña andina
}

\author{
Samir Joaqui Daza* \\ Apolinar Figueroa Casas*
}

Recibido: 25/10/2013 - Aceptado: 27/06/2014

\begin{abstract}
Resumen
El presente artículo de revisión está enmarcado en el desarrollo de una tesis doctoral en Ciencias Ambientales. Analiza los procesos tradicionales de gestión y planificación que han tenido un enfoque disciplinar para enfrentar problemáticas ambientales como el cambio climático en la alta montaña andina cuyo propósito se ha centrado en el manejo o mitigación de los impactos ambientales generados por las actividades antrópicas. Sin embargo, el deterioro ecológico aumenta debido a que las bases conceptuales en las que se fundamentan las herramientas de gestión por lo general son descontextualizadas, asumiendo que las relaciones socio ecológicas se pueden desagregar para ser estudiadas y que los fenómenos de la naturaleza son predecibles y controlables. Considerando lo anterior, se propone la incorporación de teorías complementarias como los socio-ecosistemas, la resiliencia, la transformabilidad, la adaptación y la vulnerabilidad.
\end{abstract}

Palabras clave; páramo, sistemas, resiliencia, capacidad adaptativa, ciclos de renovación adaptativa.

Biólogo, candidato a Doctor en Ciencias Ambientales de la Universidad del Cauca, trabaja en temas de resiliencia, adaptación y cambio climático en zonas de alta montaña, investigador asociado del Grupo de Estudios Ambientales, Tel +57 - 2 - 8209800 ext. 2645. Fax +57 - 2 - 8209860. Móvil: +57 - 316-4922570. Correo electrónico sjaoqui@unicauca.edu.co

** Docente titular Universidad del Cauca, Director del Doctorado en Ciencias Ambientales, Director del Grupo de Estudios Ambientales, Carrera 2-1a 25 Museo de Historia Natural. Popayán - Colombia Tel +57 - 2 - 8209800 ext. 2645. Fax +57 - 2 - 8209860. Móvil: +57 - 300-8003556. Correo electrónico apolinar@unicauca.edu.co 


\title{
Factors determining the socio-ecological resilience for andean mountains
}

\begin{abstract}
This review article is framed in the development of a doctoral thesis, it analyzes the traditional processes of management and planification which have had a disciplinary approach to cope environmental issues, such as climate change in the high mountain and whose proposal has centered on the management and mitigation of the environmental impacts generated by human activities. However, environmental degradation is increasing, due to the conceptual basis on which are based the management tools are usually decontextualized, assuming that socio ecological relationships can be disaggregated to be analyzed and that natural phenomena are predictable and controllable. Considering this, it is proposed the incorporation of complementary theories such as the socio ecosystems, the resilience, transformability and vulnerability.
\end{abstract}

Key words: Paramo, systems, resilience, adaptive capacity, adaptive renewal cycles. 


\section{INTRODUCCIÓN}

Desde el año 1500 existen fenómenos de fragmentación y cambio de uso del suelo en la alta montaña andina [1-3]; al respecto se señala que el $45 \%$ del territorio colombiano se utiliza para fines distintos a los de su vocación, y un $50 \%$ de los suelos muestra grados de erosión, porcentaje que alcanza un $80 \%$ de afectación en la región andina.

En la dinámica natural suelo-vegetación se presentan procesos como pérdida de hábitats, fertilidad del suelo, cobertura vegetal, áreas de bosque y fenómenos de fragmentación. Con relación al edafo-sistema estos procesos han generado erosión, compactación y afectación de su permeabilidad con la alteración del ciclo normal de las aguas de escorrentía superficial y subterránea[4, 5], por mencionar algunos de los efectos de las actividades antrópicas que son determinantes en la transformación de estos ecosistemas $[6,7]$.

El cambio y la variabilidad climática también contribuyen a este deterioro. El aumento promedio anual de temperatura se ha triplicado en los últimos 30 años [8-10] con lo cual se prevé la desaparición de algunos páramos $[2,11]$ y un progresivo retroceso glacial [12], entre otros fenómenos. Estas alteraciones en los rangos naturales de la variabilidad climática, que sobrepasan los umbrales que escasamente se encuentran documentados, agudizan más la preocupación por el calentamiento y las consecuencias que esto trae sobre la "integridad" de los socioecosistemas. Las afectaciones de esos cambios sobre los servicios ecosistémicos se traducen en efectos adversos sobre las comunidades humanas que están asentadas en estas regiones.

Para estos ecosistemas y en el contexto expuesto, los procesos de gestión y panificación ambiental para hacer frente a estas problemáticas han tenido un abordaje disciplinar, que enfoca acciones sobre los impactos ambientales de las actividades antrópicas. Sin embargo, el deterioro ambiental aumenta, debido a que las bases conceptuales en las que se fundamentan las herramientas de gestión por lo general son descontextualizadas, asumiendo que las relaciones de la naturaleza y la sociedad se pueden abordar por separado, y que los fenómenos de la naturaleza son predecibles y controlables [13].

\section{ANÁLISIS EN EL CONTEXTO DEL ENFOQUE SOCIOECOSISTÉMICO}

El Holoceno, que era identificado por el inicio de la agricultura, la expansión y la evolución de las distintas civilizaciones humanas durante los últimos 12 mil años, está llegando a su fin [14]. Nos enfrentamos a una nueva era con fuertes transformaciones ambientales denomina el Antropoceno, donde las alteraciones antrópicas se pueden considerar como una fuerza geológica con significativas implicaciones ambientales. 
Se ha transformado el funcionamiento del clima de la Tierra, la composición y características de los ríos, los mares y los océanos, así como la biodiversidad, el paisaje y el territorio [15].

Estos retos requieren abordar las problemáticas ambientales con perspectiva global y en contexto local, buscando o construyendo enfoques que no separen la naturaleza de la sociedad. En este sentido, dado que el hombre y la naturaleza se adaptan en un proceso de co-evolución para convertirse en un sistema integrado denominado sistema socio-ecológico o socio-ecosistema [16-18] (en adelante, SES en este texto), el análisis de las relaciones sociedad naturaleza requiere de un enfoque integrador y sistémico en un territorio con la incorporación de elementos conceptuales de los socio-ecosistemas.

Este acercamiento tiene potenciales aplicaciones y contribuciones para la gestión ambiental del territorio, ya que permite en general una comprensión integral de los conflictos e interrelaciones entre el hombre y los sistemas naturales.

Los SES se caracterizan por tres atributos que determinan su evolución: la resiliencia, la capacidad de adaptación y la transformabilidad (figura 1) [19, 20]. Aquí la resiliencia y la capacidad de adaptación se relacionan con la dinámica de un sistema, o sistemas estrechamente relacionados.

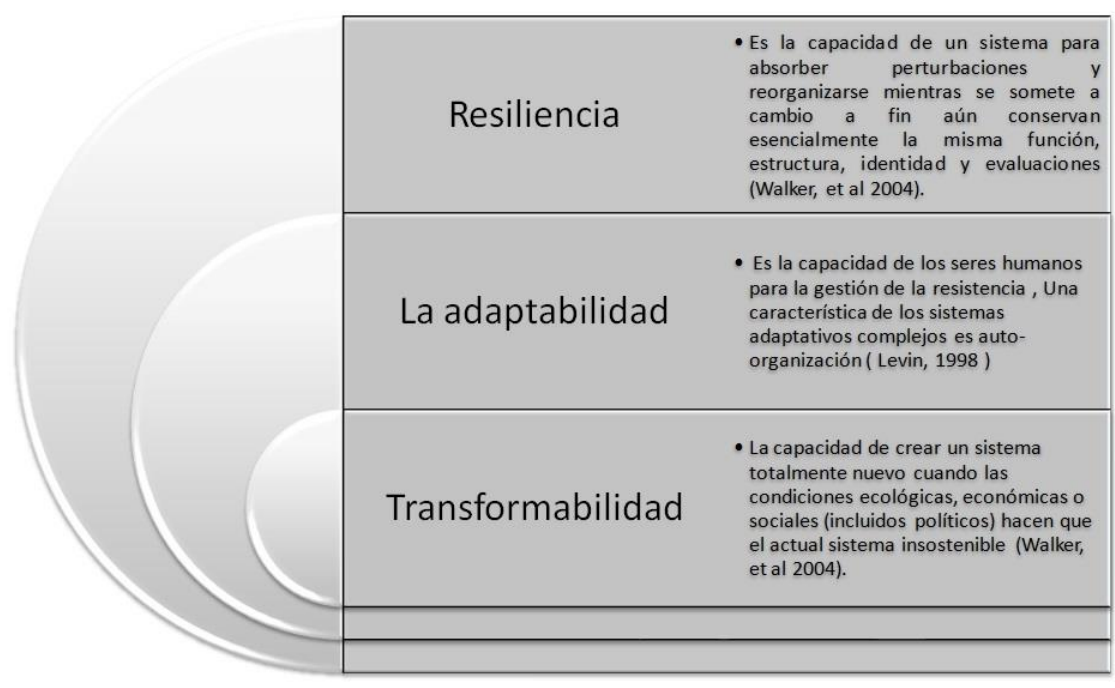

Figura 1. Atributos de los sistemas socio ecológicos.

Fuente: elaboración propia

La transformabilidad se refiere a la alteración fundamental de la naturaleza de un sistema. Este concepto aporta una visión global de la complejidad de los problemas que implican el cambio global, y sirve para tender puentes entre diversas disciplinas [21, 22]. 
Los SES se pueden abordar bajo tres perspectivas [23]: i. Como objetos de frontera, intersecciones de campos individuales de la investigación y la configuración disciplinarias; ii Como objetos epistémicos, como «cosas» que los humanos pueden y quieren saber acerca del uso de métodos bien definidos de investigación y razonamiento teórico, y iii Como objetos reales representados en los modelos de sistemas construidos para hacer frente a los problemas y fenómenos en diversos campos de aplicación.

Debido a que no existe un solo enfoque dentro del marco de los SES ni menos un planteamiento exclusivo, es necesario construir marcos de referencia teóricos (figura 2), que brinden un concepto y una definición general de los socio-ecosistemas desde la perspectiva de la complejidad; con este propósito, se exponen en este artículo los planteamientos de [24] y la influencia de la TGS, teniendo en cuenta las dinámicas de los sistemas complejos la emergencia y su consiguiente aplicación para los análisis de la vulnerabilidad, la teoría de la resiliencia, la capacidad adaptativa y la transformabilidad; de igual manera, se exponen algunos enfoques especiales para abordar los SES y otros alternativos [25-27].

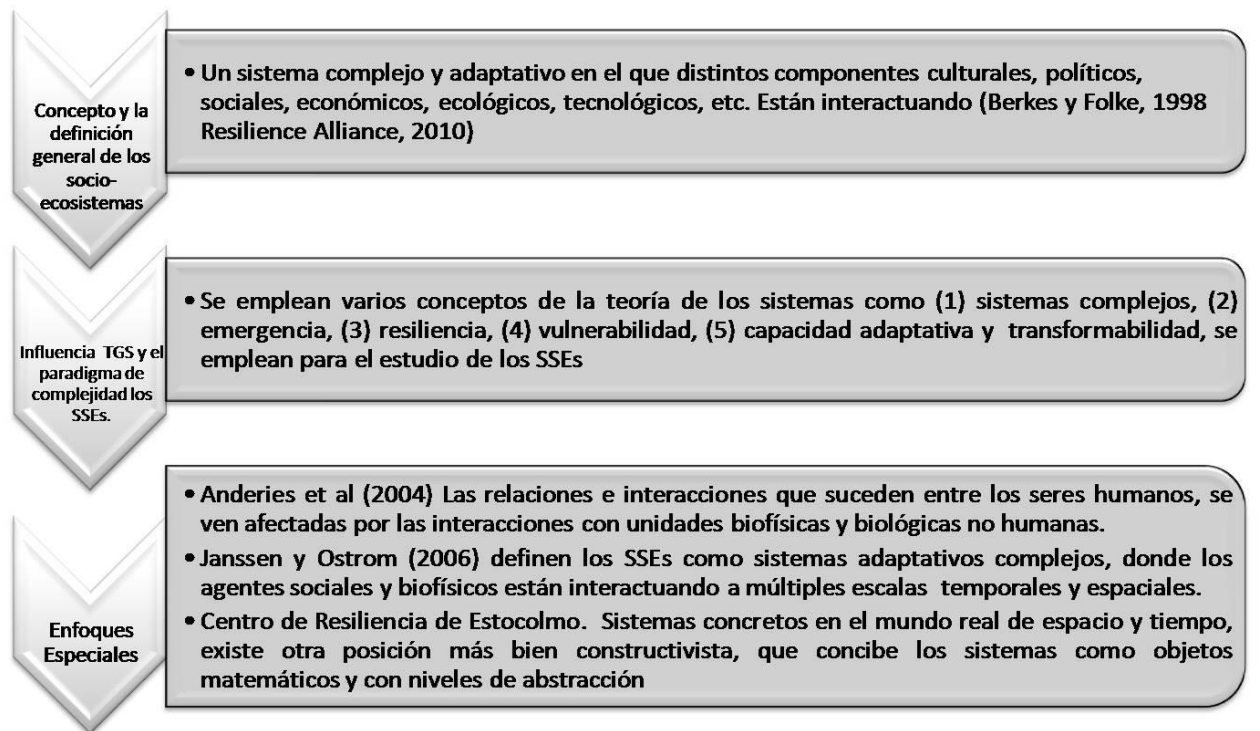

Figura 2. Marco de los SSE, enfoques teóricos. Fuente: elaboración propia

\section{TRANSFORMACIÓN, ADAPTACIÓN Y RESILIENCIA}

Tres de los procesos fundamentales que permiten estudiar, evaluar y proyectar las actividades de gestión y planificación se asocian a los siguientes aspectos: $a$ ) la trans- 
formación, entendida como el cambio que se da en las condiciones físicas, biológicas y socioeconómicas en un entorno; $b$ ) la adaptación que corresponde a la capacidad con la cual el ecosistema genera alternativas que permiten a los componentes del sistema armonizar o acoplar procesos al medio; y c) la resiliencia como la capacidad para soportar procesos de cambio, perturbación o impacto y permitir la posibilidad de recuperación de sus funciones intrínsecas vitales para la subsistencia del sistema, además de aprender del cambio y auto-organizarse.

La resiliencia, la adaptabilidad y la capacidad de transformación, más que atributos que determinan la trayectoria de los SES, son las dimensiones, pues los atributos son individuales, y los sistemas son más que la suma de un conjunto de unidades relacionadas. Los SES son ecosistemas que de una forma compleja se vinculan e interaccionan de manera dinámica e interdependiente con uno o más sistemas sociales.

Abordar las problemáticas ambientales desde la perspectiva de los socio-ecosistemas [28-32] requiere que se consideren en términos de su estructura y dinámica. Dado que el abordaje tradicional sobre lo comprensión científica, y sobre todo la comprensión ecológica se han enfocado sobre análisis biofísicos, y reconociendo que este abordaje es importante para hacer frente a los problemas ambientales, hay que aceptar que el conocimiento científico difícilmente será suficiente [33]. Lo anterior conduce a que los procesos de influencia humana sobre los componentes del sistema (flora, fauna, suelo y agua) se solucionen con la comprensión de los procesos biofísicos sobre los que se toman decisiones, para hacer las herramientas de planificación más objetivas. Sin embargo, esta visión tiene un alto grado de incertidumbre.

En términos de las ciencias ambientales la incertidumbre es constante, dado que los estudios técnicos de la naturaleza se realizan por componentes y esporádicamente se analizan sistémicamente. Además, el análisis del acoplamiento ser humano-naturaleza es muy poco abordado, considerando que la indeterminación y la complejidad se convierten en constantes que se deben sortear. Es importante reconocer que lo tradicional no funciona y replantear los problemas, y analizar la naturaleza y el ser humano bajo un enfoque integrador, y no como entidades independientes en una relación de sujeto-objeto de investigación, conceptualización requiere la vinculación de diversas disciplinas.

El análisis de la naturaleza y el ser humano debería orientarse hacia el entendimiento de la relación de la naturaleza y la sociedad en términos de sus procesos, como los vínculos que han existido entre la sociedad y el ambiente. Más que establecer relaciones disciplinares que tradicionalmente analizan por separado estas dos entidades, este abordaje es pertinente, ya que permitiría configurar herramientas de gestión que aporten realmente a la solución de las problemáticas ambientales. 
Lo anterior demanda que en lugar de preguntar cómo la sociedad puede mejorar o cómo se puede hacer mejor gestión de los recursos ecológicos, debería orientarse a cómo se hace que los SES sean más robustos [17]; sin embargo, la robustez refiere al mantenimiento del rendimiento del sistema cuando es sometido a perturbaciones externas e impredecibles, lo cual aplica para sistemas controlables desde la ingeniería, pero en la naturaleza es difícil controlar o predecir el comportamiento; se puede reducir la incertidumbre pero no controlar los procesos.

\section{ENFOQUE DE RESILIENCIA}

Por otro lado el abordaje desde la resiliencia socioecosistémica con herramientas de gestión, y entendiéndola como la capacidad que tienen los sistemas de resistir una perturbación, recuperarse y auto-organizarse [34-36] puede contribuir al entendimiento de las relaciones de ser humano con la naturaleza, trabajando en la recuperación de las funciones ecosistémicas, más que en procesos de conservación.

La visión de resiliencia socioecosistémica se convierte una herramienta clave para la gestión, vinculando el conocimiento de los procesos que determinan el comportamiento del sistema sin ejercer acciones en los efectos de las presiones antrópicas sobre los SES.

Cuantificar y mesurar la capacidad de resiliencia de un sistema ante una perturbación, podría abrir importantes caminos para la comprensión de los ciclos adaptativos de los sistemas [20,37] y contribuir a la reducción de los espacios que existen en las brechas conceptuales para la comprensión de la resiliencia y la adaptación en el marco de los socioecosistemas de la alta montaña de los Andes del norte.

La resiliencia ha tenido dos abordajes en la ecología. El primero, centrado en la recuperación del sistema y el tiempo de retorno después de una perturbación, y el segundo, centrado en la cantidad de perturbaciones que un sistema puede asimilar sin cambiar su función [38]. En los últimos años la resiliencia ha ampliado su espectro a los socio-ecosistemas, enfatizando en tres elementos fundamentales: la persistencia, la adaptabilidad y la transformabilidad [39]. Esta ampliación del concepto de resiliencia ha traído dentro de su ámbito el papel de las instituciones, el capital social, el liderazgo y el aprendizaje.

Teniendo en cuenta que los SES pasan por ciclos irregulares de organización, crecimiento, colapso y renovación [40], para interpretar la dinámica de un sistema particular, desde su gestión, conservación y manejo, se deben definir con claridad las condiciones de la resiliencia.Esta elasticidad se mueve por espacios multidimensionales que son controlados por diferentes procesos; los cambios que se presentan en los 
ecosistemas se miden en escalas espaciales y temporales, y están sujetos a aspectos sociales, económicos, productivos, entre otros.

Un aporte significativo para comprender las propiedades no lineales de los socioecosistemas son los ciclos de renovación adaptativa de un sistema [37, 40]; este aporte se construye en atención a que los sistemas naturales y sociales en su funcionamiento no son lineales; esta teoría busca comprender esta propiedad, la no- linealidad de los sistemas complejos socioecológicos; un posterior desarrollo que complementa y amplía las dinámicas del ciclo adaptativo está contenido en el concepto de "Panarquía"[40] desarrollado por Holling y Gunderson que contempla las dinámicas del ciclo adaptativo especialmente en aquellos sistemas socio-ecológicos donde la interacción y el desarrollo de la sociedad con su entorno son lo más importante.

El concepto de resiliencia parte del reconocimiento del cambio continuo de los sistemas, como estrategia para gestionar la capacidad que tienen los SES de hacer frente, adaptarse y cambiar [34]. La gestión de la resiliencia implica el mantenimiento de opciones para un entorno que cambia rápidamente, donde la sorpresa es constante, enfrentando un futuro impredecible, por lo que la resiliencia tiene una mirada hacia el futuro [41]. La resiliencia y la vulnerabilidad tienen significados opuestos, pero complementarios [34]: en un sistema resiliente, el cambio tiene el potencial de crear oportunidades para el desarrollo, la novedad y la innovación, y para auto-organizarse; en un sistema vulnerable, incluso los pequeños cambios pueden implicar importantes cambios.

En la evaluación de la resiliencia de un sistema, se debe especificar la configuración del sistema y qué perturbaciones son de interés [42], para abordar las siguientes tres características generales :i)La cantidad de cambio o transformaciones que un sistema complejo puede soportar manteniendo las mismas propiedades funcionales y estructurales, ii) el grado en el que el sistema es capaz de autoorganizarse y iii) la habilidad del sistema complejo para desarrollar e incrementar la capacidad de aprender, innovar y adaptarse.

Es necesario promover la creación de nuevos modelos de investigación interdisciplinarios que contribuyan a romper la clásica división (conceptual y metodológica) entre las ciencias sociales y las ciencias biofísicas, y que permitan tender sólidos puentes entre los investigadores (generadores de conocimiento) y los gestores y tomadores de decisiones. La capacidad de transformación es una característica fundamental en los sistemas sustentables (sociales, ecológicos, políticos, económicos o tecnológicos); sin embargo, el aspecto que más incidencia tiene para el humano es que estos sistemas deben presentar una fuerte predisposición a la innovación y el aprendizaje; es decir, debe existir evolución evidente. 


\section{REFERENCIAS}

[1] D. Armenteras et al., "Andean forest fragmentation and the representativeness of protected natural areas in the eastern Andes, Colombia," Biological Conservation, vol. 113, no. 2, pp. 245-256, 2003.

[2] N.E. Rodríguez et al., Cambio climático y su relación con el uso del suelo en los Andes colombianos. Bogotá D.C, Instituto de Recursos Biológicos Alexander von Humboldt 2010, 80 p.

[3] A. Etter et al., "Regional patterns of agricultural land use and deforestation in Colombia," Agriculture, Ecosystems \& Environment, vol. 114, no. 2-4, pp. 369-386, 2006.

[4] J. Martínez et al., "Cambios de cobertura y fragmentación a través de un análisis espacio temporal en el parque nacional natural Puracé", en Fragmentación y coberturas vegetales en ecosistemas andinos, departamento del Cauca., A. Figueroa y M. Valencia,“eds»., pp. 137-155, Popayán: Universidad del Cauca, 2009.

[5] S. Joaqui y A. Figueroa, «Análisis multitemporal de coberturas vegetales para ecotopos paramunos. parque nacional natural Puracé»,en Fragmentación y coberturas vegetales en ecosistemas andinos, departamento del Cauca., A. Figueroa y M. Valencia, “eds»., pp. 119135, Popayán: Universidad del Cauca, 2009.

[6] G. Márquez, "Ecosistemas Estrategicos de Colombia" Revista de la Sociedad Geográfica de Colombia,volume. 46, no.135, pp. 87-103, 2003

[7] O. Rangel, "Biodiversidad en la región del páramo: con especial referencia a Colombia", presentado en Congreso Mundial de Páramos, Paipa-Boyaca, 2002.

[8] M. Vuille, y R. S. Bradley, "Mean annual temperature trends and their vertical structure in the tropical Andes" Geophys. Res. Lett, vol. 27, no. 23, pp. 3885-3888, 2000.

[9] H. Gutiérrez "Modelo para evaluar la vulnerabilidad de las coberturas vegetales de Colombia ante un posible cambio climático utilizando SIG con énfasis en las coberturas nival y de páramo", Bogotá, pp.122-131,2002.

[10] S. K. Herzog et al., Cambio climático y biodiversidad en los Andes tropicales, Instituto Interamericano para la Investigación del cambio global (IAI), Sao José dos Campos y Comité Científico sobre Problemas del Medio Ambiente (SCOPE). Paris, 2012, 426 p.

[11] W. Buytaert et al., "Human impact on the hydrology of the Andean páramos",Earth-Science Reviews, vol. 79, no. 1-2, pp. 53-72, 2006.

[12] G. Poveda, y K. Pineda, "Reassessment of Colombia's tropical glaciers retreat rates: are they bound to disappear during the 2010-2020 decade?",Advances in Geosciences, vol. 22, pp. 107-116, 2009.

[13] S. Farhad, Los sistemas socio-ecológicos. Una aproximación conceptual y metodológica, Reporte Universidad Pablo de Olavide, Sevilla, España, 2012. 
[14] R. Fernández Durán, «El Antropoceno: la crisis ecológica se hace mundial. La expansión del capitalismo global choca con la biosfera». Madrid: Coeditores. Virus y Libros en Acción, 2010.

[15] M. Davis, “Bienvenidos al Antropoceno,’[En linea],Revista Digital Sinpermiso,Disponible:http:// www.sinpermiso.info/textos/index.php?id=1954, 2008.

[16] B. Martín-López et al., "Un marco conceptual para la gestión de las interacciones naturalezasociedad en un mundo cambiante",Cuides, vol. 3, pp. 229-258, 2006.

[17] J. Anderies et al., "A Framework to Analyze the Robustness of Social-ecological Systems from an Institutional Perspective",Ecology and Society, vol. 9, no. 18, p. 17, 2004.

[18] G. C. Gallopín, “Linkages between vulnerability, resilience, and adaptive capacity”,Global Environmental Change, vol. 16, no 3, pp. 293-303, 2006.

[19] C. Folke et al., "Resilience thinking: integrating resilience, adaptability and transformability",Ecology and Society, vol. 15, no. 4, pp. 20, 2010.

[20] B. Walker, y D. Salt, Resilience Thinking Sustaining Ecosystems and People in a Changing World, Washington DC,Island Press ed., 2006, p 174.

[21] Fundacíon IPADE, Cuatro grandes retos, una solución global: Biodiversidad, cambio climático, desertificación y luch a contra la pobreza,J. Gonzales. e I. Santos,(eds),Madrid: Fundación IPADE y Agencia Española de Cooperación para el Desarrollo- AECID, 2011,302 p.

[22] C. Duarte et al., Cambio global Impacto de la actividad humana sobre el sistema Tierra, Madrid:Consejo Superior de Investigaciones Cientificas, 2006, 167 p.

[23] E. Becker, "Social-ecological systems as epistemic objects" Human-Nature Interactions in the Anthropocene: Potentials of Social-Ecological Systems Analysis. London: Routledge, pp. 37-59, 2012.

[24] F. Berkes, y C. Folke, "Linking social and ecological systems for resilience and sustainability" Linking social and ecological systems: management practices and social mechanisms for building resilience, pp. 1-25, 1998.

[25] L. Von Bertalanffy, Teoría general de los sistemas: edição comemorativa: Fondo de Cultura Económica, 2006, 353 p.

[26] J. M. Anderies et al., "A framework to analyze the robustness of social-ecological systems from an institutional perspective",Ecology and Society, vol. 9, no. 1, p. 18, 2004.

[27] ResilienceAlliance, Assessing and managing resilience in social-ecological systems: A practitioners workbook Version $1.02007,84$ p.

[28] J. M. Alier, “Conflictos ecológicos y justicia ambiental,” PapelesdeRelaciones Ecosociales y Cambio Global, Vol. 103, pp. 11-28, 2008.

[29] G. Peterson et al., "Ecological Resilience, Biodiversity, and Scale”,Ecosystems, vol. 1, no. 1, pp. 6-18, 1998. 
[30] C. Folke, "Resilience: The emergence of a perspective for social-ecological systems analyses",Global Environmental Change vol. 16, pp. 15, 2006.

[31] Q. Vilardy, “Estructura y dinámica de la ecorregión Ciénaga Grande de Santa Marta: una aproximación desde el marco conceptual de los sistemas socio-ecológicos complejos y la teoría de la resiliencia”, tesis de Facultad de Ciencias Departamento Interuniversitario de Ecología, Universidad Autónoma de Madrid, Madrid, 2009.

[32] C. S. Holling, "Understanding the Complexity of Economic, Ecological, and Social Systems",Ecosystems vol. 4, pp. 4, 2001.

[33] D. Ludwig et al., "Ecology, conservation, and public policy," Annual Review of Ecology and Systematics, pp. 481-517, 2001.

[34] C. Folke et al., "Resilience and sustainable development: building adaptive capacity in a world of transformations", AMBIO: A Journal of the Human Environment, vol. 31, no. 5, pp. 437-440, 2002.

[35] C. S. Holling, "Resilience and Stability of Ecological Systems", Annual Review of Ecology and Systematics, vol. 4, pp. 23, 1973.

[36] L. H. Gunderson, "Ecological Resilience--In Theory and Application", Annual Review of Ecology and Systematics, vol. 31, pp. 425-439, 2000.

[37] S. Carpenter et al., "From Metaphor to Measurement: Resilience of What to What?," Ecosystems, vol. 4, no. 8, pp. 765-781, 2001.

[38] F. Miller et al., "Resilience and Vulnerability: Complementary or Conflicting Concepts?," Ecology and Society, vol. 15, no. 3, pp. 11, 2010.

[39] N. M. Gotts, "Resilience, panarchy, and world-systems analysis," Ecology and Society, vol. 12, no. 1, pp. 24, 2007.

[40] C. S. Holling, "Understanding the Complexity of Economic, Ecological, and Social Systems," Ecosystems, vol. 4, no. 5, pp. 390-405, 2001.

[41] F. Berkes, y C. S. Seixas, "Building Resilience in Lagoon Social-Ecological Systems: A LocalLevel Perspective," Ecosystems, vol. 8, no. 8, pp. 967-974, 2005.

[42] A. Calvente, Resiliencia: un concepto clave para la sustentabilidad, Reporte, Universidad Abierta Interamericana, Buenos Aires, 2007. 